\title{
Crystal structures of Streptomyces coelicolor methylmalonyl-CoA epi- merase with substrate or transition state analog contradicts a simple general acid-base catalytic mechanism
}

\author{
Lee M. Stunkard, Aaron B. Benjamin, James B. Bower, Tyler J. Huth and Jeremy R. Lohman* \\ Department of Biochemistry, Purdue University Center for Cancer Research, Purdue University, West Lafayette, Indiana \\ 47907, United States
}

Supporting Information Placeholder

\begin{abstract}
Isomerases that flip carbon stereocenter chirality often use straightforward general acid base catalysis where a proton is abstracted from one face of a central carbon and a proton is deposited on the opposite. That mechanism has been ascribed to methylmalonyl-CoA epimerase (MMCE). The proposed mechanism is use of concerted general acid-base catalysis similar to the proline racemase, based on the exchange of the $\alpha$-carbon proton with solvent. Tritium kinetic isotope effect experiments lead to the hypothesis of a "protected base" in catalysis. Structures of Propionibacterium shermanii MMCE with catalytic $\mathrm{Co}^{2+}$ bound revealed that two glutamates were positioned on opposite sides of the active site, that could fulfil the roles of the general acids. However, in preliminary modeling of the substrate, we noticed the distances and geometry were not typical suggesting conformational changes upon substrate binding. In order to further understand the puzzling relationships between the catalytic mechanism, substrate preference and structure, we solved MMCE structures with the substrate and a transition state analog bound. Our structures reveal that there are no acids or bases near the $\alpha$-carbon for simultaneous deprotonation/reprotonation. Therefore, we propose two alternatives for MMCE catalysis, one where conformational changes and water invasion overcome the long $\mathrm{C} 2$-catalytic acid distances observed in the crystal structures. Alternatively, a typically disallowed 1,3-sigmatrophic hydrogen shift results in enolization, whereby the thioester ketone or carboxylate acts as the "protected base". Both mechanisms are compatible with other 2-substituted malonyl-CoAs, as such our structures provide a platform to design mutations for expanding substrate scope to support combinatorial biosynthesis.
\end{abstract}

Methylmalonyl-CoA epimerase (MMCE) or racemase is central to linking TCA cycle intermediates with other metabolic pathways in various organisms, including humans. ${ }^{1}$ Typically, MMCEs are found with methylmalonyl-CoA mutases, where the mutase reversibly converts succinyl-CoA to $(2 R)$-methylmalonyl-CoA (L-configuration) and the epimerase generates (2S)-methylmalonyl-CoA (Dconfiguration). Some organisms like Escherichia coli and Pseudomonas putida are lacking a MMCE. ${ }^{2}$ In order to generate $E$. coli capable of supporting the biosynthesis of polyketides like erythromycin, Streptomyces coelicolor MMCE (ScMMCE) was introduced to link the production of $(2 R)$-methylmalonyl-CoA from the activity of the native methylmalonyl-CoA mutase (Sbm) to the (2S)-methylmalonyl-CoA specific 6-deoxyerythronolide B synthase (DEBS). ${ }^{3-4}$ This establishes MMCE as important not only in intermediary metabolism, but also for the production of polyketides in certain heterologous hosts.

Another source of methylmalonyl-CoA for polyketide biosynthesis is through the activity of malonyl-CoA synthetases such as MatB from S. coelicolor or Rhizobium trifolii although they generate $(2 R)$-methylmalonyl-CoA thus also requiring MMCE to support efficient DEBS-like polyketide biosynthesis. ${ }^{5-6}$ MatB enzymes also accept substrates other than malonate and methylmalonate with poor efficiency, ${ }^{7}$ although mutants have been generated with expanded substrate specificity. ${ }^{8}$ Therefore, an attractive path to polyketide derivatives is through the engineering of MatB to produce malonyl-CoA with novel 2-substitutents combined with acyltransferases engineered to accept the novel malonyl-CoAs. ${ }^{9-14}$ However, it is likely the engineered enzymes generate substrates that are not directly accepted by the acyltransferases, requiring spontaneous epimerization which has a half-life of $\sim 90$ hours for methylmalonyl-CoA at $\sim \mathrm{pH} 7$ and $0{ }^{\circ} \mathrm{C} .{ }^{15}$ Therefore, MMCE enzymes are likely a necessary addition in the engineered production of polyketides through MatB mutants, supporting further characterization of MMCE structure-function relationships.

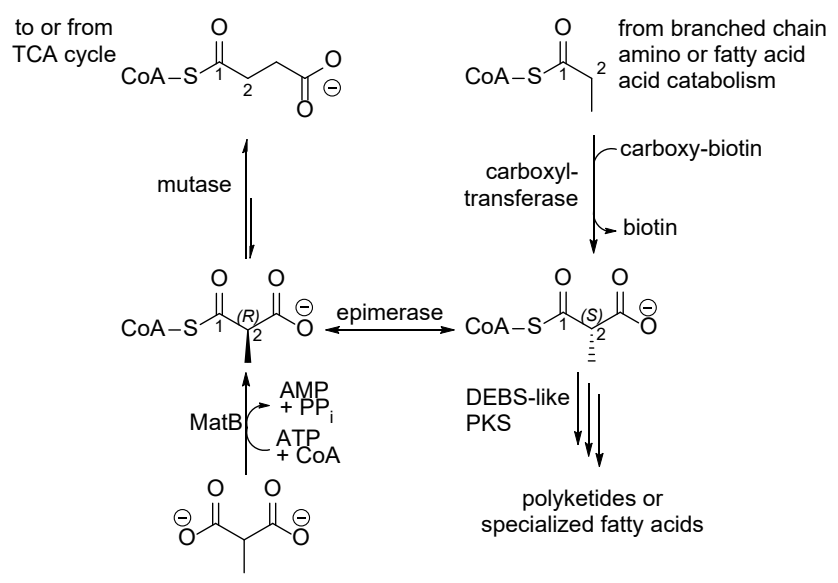

Figure 1. MMCE is central to the metabolism of methylmalonylCoA by linking enzymes with different stereochemical preference or outcomes.

The catalytic mechanism of Propionibacterium shermanii MMCE (PsMMCE) was probed using hydrogen-tritium exchange assays. ${ }^{15-16}$ In both the tritium exchange assays and an NMR study, ${ }^{17}$ PsMMCE catalyzes exchange of the $\mathrm{C} 2$ hydrogen-isotope 
with solvent-isotopes, which was the first piece of evidence suggesting a general acid-base catalysis mechanism, Figure 2 . In the tritium exchange assays, in which only the $(2 R) \rightarrow(2 S)$ direction was followed, it was determined the enzyme essentially always exchanges the $\mathrm{C} 2$ hydrogen-isotope with solvent during catalysis and the 2-hydrogen isotope is not exchanged with solvent or "protected" until product release. However, there was a low kinetic isotope effect reported suggesting other factors rather than chemistry are rate limiting, such as conformational changes or product release. Substrate bound structures of the enzyme could provide insight into those other factors.

Structures of PsMMCE in the apo and $\mathrm{Co}^{2+}$ bound holoforms, revealed an active site with partially conserved glutamate residues on opposing sides of the active site. ${ }^{18}$ Based on modelling of the $(2 R)$-epimer, it was predicted that the glutamates acted as the catalytic acid-base pair. In order to clarify the roles of the proposed catalytic residues and gain insight into enzyme-substrate interactions for expanding substrate scope, we solved the structure of ScMMCE with methylmalonyl-CoA and a putative transition state analog, 2-nitropropionyl-CoA, Figure 3. We previously synthesized the nitro bearing methylmalonyl-CoA analog for examining the structure-function activities of various enzymes. In the active site of E. coli methylmalonyl-CoA decarboxylase and a bifunctional acyltransferase/decarboxylase, LnmK, the nitro analog binds as a nitronate with a deprotonated $\mathrm{C} 2 .{ }^{19-20} \mathrm{We}$ expected 2-nitropropionyl-CoA to be an ideal transition state analog to reveal interactions with the catalytic acids.

$$
\begin{aligned}
& \text { A } \\
& \text { B }
\end{aligned}
$$

Figure 2. Two possible catalytic mechanisms for MMCE. A) Previously proposed general acid-base catalysis. B) Enolization via 1,3-H shift with solvent tautomerization.
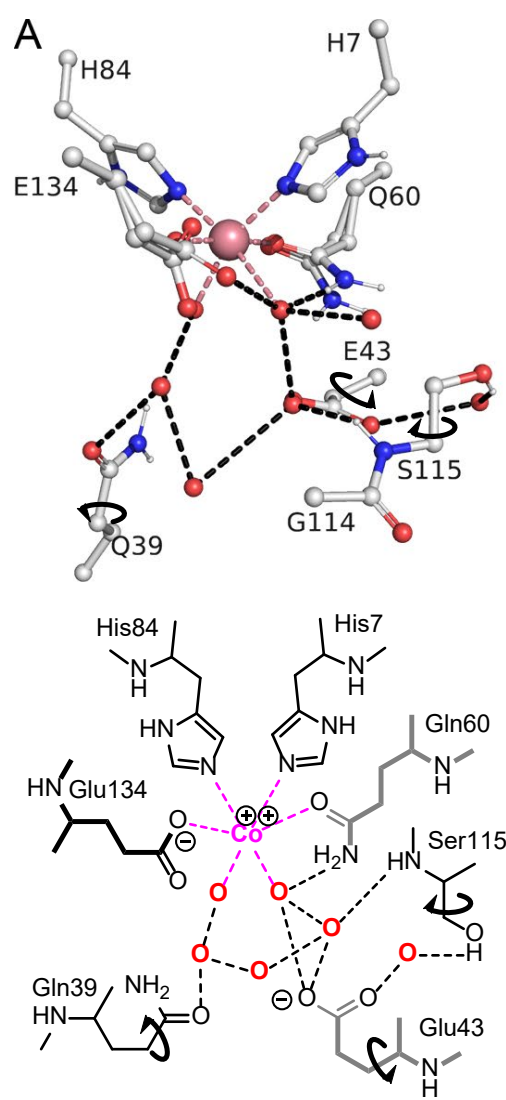
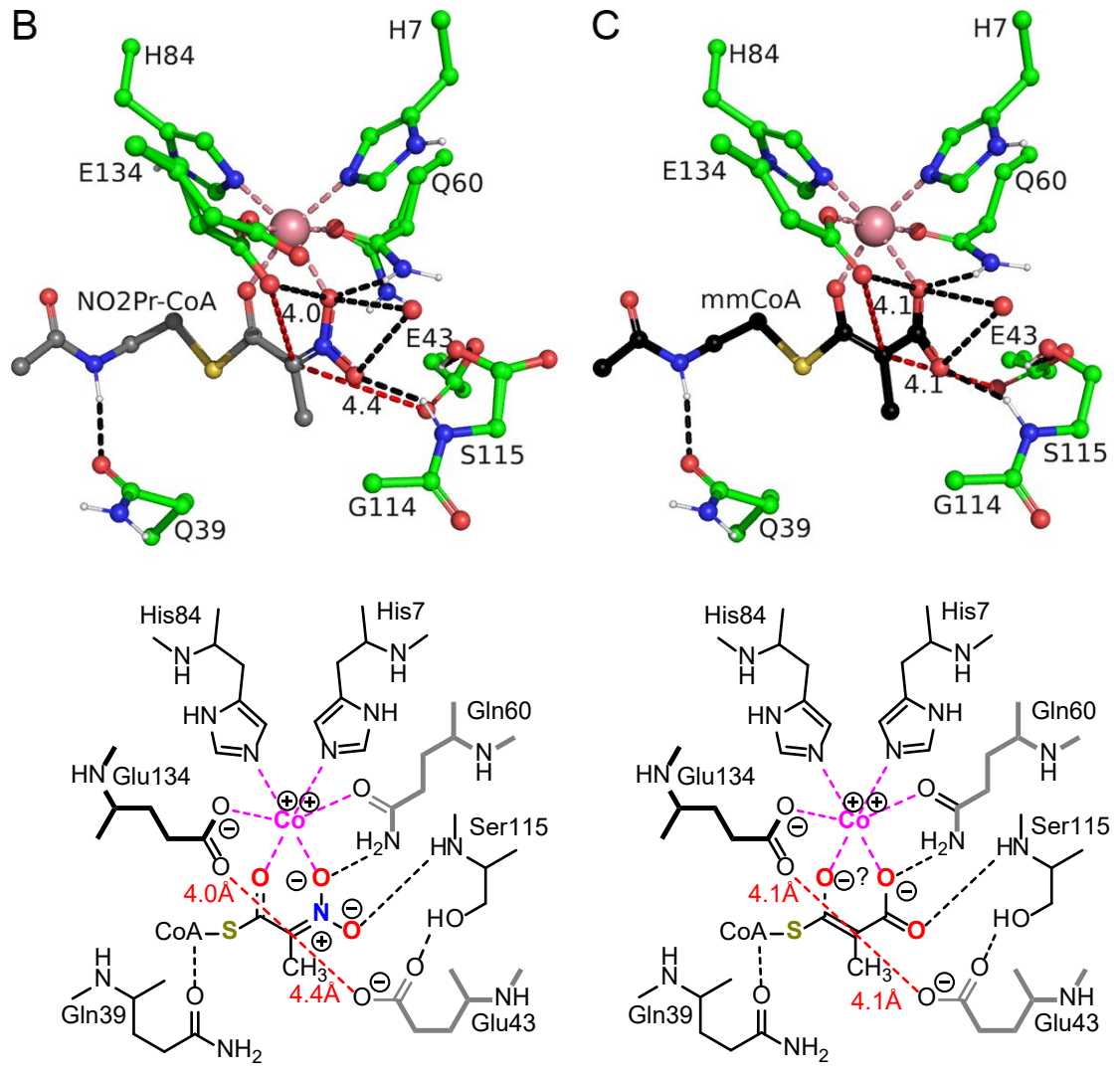

Figure 3. Active sites of ScMMCE as the holoenzyme and ligand bound states with flattened schematics. Hydrogens are shown as diminutive ball and sticks. Black dashes represent potential hydrogen bonds or close interactions. Red dashes represent distances to predicted catalytic acids. A) Holoenzyme, bold arrows represent conformational changes necessary for ligand binding. Hydrogens are not shown on the active site waters shown as red "O" in the flattened schematic. B) 2-nitronate-propionyl-CoA $\left(\mathrm{NO}_{2} \mathrm{Pr}-\mathrm{CoA}\right)$ is shown as gray sticks. C) Methylmalonyl-CoA is shown as black sticks. 

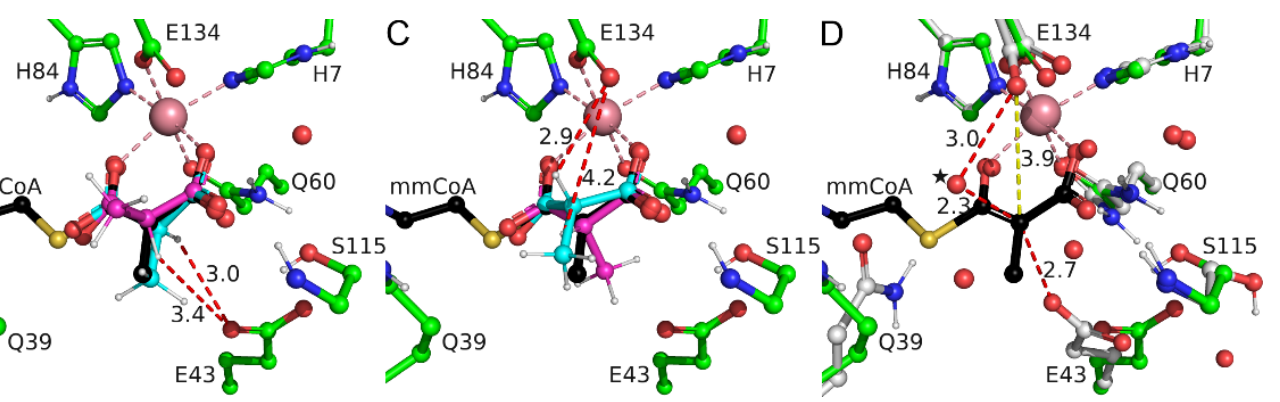

Figure 4. Active site of holo-ScMMCE with substrate bound. Hydrogens are shown as diminutive ball and sticks. A) Electron density for the methylmalonyl-thioester portion of methylmalonyl-CoA in the ScMMCE active site displayed as sigma-A weighted $2 \mathrm{mF}_{\mathrm{o}}-\mathrm{DF} \mathrm{F}_{\mathrm{c}} \mathrm{maps}$ displayed $1.0 \sigma$ (blue mesh) and $2.0 \sigma$ (cyan mesh). B) Overlay of methylmalonate- $\mathrm{Co}^{2+}$ complex (CSD JERMAU) on the methylmalonylthioester- $\mathrm{Co}^{2+}$ of holo-ScMMCE representing the $(2 R)$ configuration. The structure JERMAU has two overlapping configurations, one where the methyl-group and non- $\mathrm{Co}^{2+}$ coordinating oxygens are coplanar designated in cyan, and the other where the methyl group is equatorial designated in magenta. C) Similar to panel B but representing the $(2 S)$ configuration. D) Overlay of holo-ScMMCE without substrate and with the methylmalonyl-CoA enol/enolate bound, coloring same as Figure 3. The water indicated by a star from holo-MMCE without substrate bound makes a close contact with $\mathrm{C} 2$ of the enol/enolate intermediate and would be activated by Glu 134 , indicated by red dashes. The conformation of Glu43 from holo-MMCE without substrate configuration also makes a close contact with the substrate $\mathrm{C} 2$ shown as red dashes.

We solved the structure of holo-ScMMCE in order to compare with the structures of other MMCE homologs. Crystals were grown at neutral $\mathrm{pH}$ with polyethylene glycol as the precipitant, which diffract to $\sim 1.4 \AA$ resolution, data collection and refinement statistics in Table S1. The asymmetric unit contains a dimer which is expected to be relevant in solution based on buried surface area and homology with other MMCEs, Figure S1. ${ }^{21}$ There is clear evidence for a metal in both active sites with octahedral coordination, Figure $3 \mathrm{~A}$ and Figure S2. While the metal can't be unambiguously assigned as a $\mathrm{Co}^{2+}$, since the protein was produced with trace metals supplemented into the media, $\mathrm{Co}^{2+}$ has appropriate distances and coordination geometry. The active site of chain A has multiple conformations for Gln60 and Glu134, while the active site of chain B has multiple conformations for Gln39 and Gln60. The monomers have slight differences in the positions of active site residues Gln39, Glu43, and Ser115, Figure S2. An active site loop with residues 37-41 in chain B has two conformations. The alternative conformations in the active site suggests two different protonation states or hydrogen bonding patterns of the waters interacting with $\mathrm{Co}^{2+}$, Glu43 or Glu134.

Comparison of holo-ScMMCE with other MMCE structures reveals two major clades with distinct active site residues. A DALI comparison with PsMMCE (PDB 1JC5) reveals an overall sequence identity of $42 \%$ with an RMSD of $1.5 \AA$ over 137 residues. The active site $\mathrm{Co}^{2+}$ coordinating residues and predicted catalytic residues are conserved, Figure S1. Comparison of holo-ScMMCE with human mitochondrial MMCE (HsMMCE, PDB 3RMU) reveals an overall sequence identity of $34 \%$ with an RMSD of $2.0 \AA$ over 131 residues. ${ }^{22}$ The active sites of ScMMCE and HsMMCE differ with respect to one of the catalytic acids Glu43 being a hydrophobic Val86 and the $\mathrm{Co}^{2+}$ coordinating Gln60 being an acidic Glu97, Figure S1. Furthermore, Glu97 doesn't directly coordinate $\mathrm{Co}^{2+}$, rather coordination is via a water. The differences in active sites suggests these two clades may have different catalytic strategies for proton abstraction and donation.

We solved the structure of ScMMCE with 2-nitropropionylCoA, which should be an ideal transition state analog for general acid-base catalysis. Hydrolysis of acyl-CoAs during crystallization is problematic, thus we chose to screen conditions using our hydrolysis stable substrate analog 2-nitropropionyl-amino(dethia)CoA, which yielded crystals in a number of conditions. We optimized crystallization of ScMMCE with 2-nitropropionylamino(dethia)CoA, which grew well-diffracting crystals from high ammonium sulfate at neutral $\mathrm{pH}$ within 48 hours. Crystals diffracted to $1.37 \AA$, Table S1. The spacegroup is different than holo-
ScMMCE and the asymmetric unit only has a monomer. There is clear electron density for 2-nitropropionyl-CoA and a metal such as the modeled $\mathrm{Co}^{2+}$, Figure S3. However, there is a small amount of negative electron density for the thioester sulfur and $\mathrm{Co}^{2+}$, suggesting analog and metal are close to, but not fully occupied. Nevertheless, the 2-nitropropionyl-CoA electron density reveals the transition state analog binds as the planar nitronate, which mimics a planar enolate intermediate, Figure 3B. The active site residues Gln60, Ser115 and Glu134 have multiple conformations. The distances between the 2-nitropropionyl-CoA C2 and potential catalytic acids Glu43 and Glu134 are $4 \AA$ and $4.4 \AA$. While an essentially perfect transition state analog is bound, the long distances and geometry are incongruent with a catalytic state. This prompted us to pursue structures with the natural substrate.

Based on the refined crystallization conditions for the transition state analog, we generated a co-crystal structure with $(2 R S)$ methylmalonyl-CoA. The crystals diffracted well with good statistics, Table S1. However, the electron density for the metal coordination site was only appropriate for an atom with 18 electrons such as water or ammonium, which was used as the crystallization precipitant, Figure S3. The modeled ammonium is accompanied by poor electron density for the methylmalonyl-thioester of methylmalonyl-CoA, which we have modeled in two conformations reflecting $2 R$ - and $2 S$-bound states. There is some likely hydrolysis as reflected by a lack of electron density for the methylmalonyl $\mathrm{C} 2$, with oxygen coordinating sites at close to full occupancy due to binding of water as in the holo-ScMMCE active site. The similar crystallization conditions between substrate and transition state analog suggest that 2-nitropropionyl-CoA forms a more tightly bound enzyme-metal-ligand complex than substrate, since $\mathrm{Co}^{2+}$ is readily displaced by a high concentration of ammonium with substrate present, but not the analog.

Addition of $1 \mathrm{mM} \mathrm{CoCl}_{2}$ in the crystallization condition for ScMMCE with methylmalonyl-CoA produced crystals diffracting to $1.8 \AA$, Table S1. Addition of cobalt to the crystallization condition leads to full occupancy in the metal coordination site, Figure S4. Electron density is strong for every heavy atom of the substrate, except the methyl group of methylmalonyl-thioester, which is weak in omit maps, Figure S4C. Refinement as any of the $2 R, 2 S$, or enol/enolate intermediate leads to electron density in a position resembling the enol/enolate intermediate state, where the methyl group is in plane with the thioester and carboxylate, Figure 4A. Models for the $(2 R)$ and $(2 S)$ states based on structures of methylmalonate- $\mathrm{Co}^{2+}$ complex (CSD JERMAU or JERMEY) ${ }^{23}$ are 
shown in Figure 4B and 4C. An average of the methyl-group positions found in Figure 4B and 4C can explain the weak electron density. The active site residues in the methylmalonyl-CoA/ $\mathrm{Co}^{2+}$ bound state are found as single conformations. The single conformations compared to the transition state analog bound state may be reflective of differences in resolution. Nevertheless, both the transition state analog and substrate enol/enolate models reveal the proposed catalytic acids are too far from the substrate to perform their predicted roles.

A comparison of the holo-ScMMCE structures with and without ligands reveal relatively large conformational changes for a number of active site residues including Gln39, Glu43 and Ser115, and essentially removal of all active site waters, except one that is in direct contact with bulk solvent, Figure 3. In the ligand free state, Glu43, the predicted catalytic acid is positioned correctly for proton abstraction/donation from/to the $2 R$ face of the enolate intermediate, Figure $4 \mathrm{D}$. On the opposite side of the active site, proton abstraction/donation from/to the $2 S$ face of the intermediate could be accomplished by a water molecule bridging Glu134 and C2, that is also observed in the ligand free structures. This suggests that conformational changes and water invading the active site might be a catalytic strategy used by ScMMCE and homologs in the clade.

Introduction of water into the enolate bound active site still poses a problem, as protonation of the substrate enolate would lead to a hydroxide being formed likely in the presence of a negatively charged carboxylate. Alternatively, a typically disallowed 1,3-sigmatrophic hydrogen shift moves the proton onto the thioester ketone or carboxylate, Figure 2B. Protonation of the thioester ketone or carboxylate would generate a species that has less affinity for the cobalt, whereby the proton may displace the oxygen ligands from the metal allowing disassociation. In solution the enol can be resolved by water generating either $(2 S)$ - or $(2 R)$-product. For the clade represented by $\mathrm{HsMMCE}$, the residue corresponding to Glu134 is a valine, which removes a catalytic acid. As such, the enolization model is attractive for the HsMMCE clade as it removes the need for a catalytic acids and bases.

The active site surrounding the methyl group of the enol/enolate intermediate is made of Asn36, Gln39, Val41 and Ile120. There is some space to accommodate a larger ethylmalonyl-thioester substrate, but not likely any larger. Mutation of Val41, Ile120 and maybe Asn36 are expected to open the active site to larger substrates without deleterious effect. Such mutant ScMMCE enzymes are likely to support engineering efforts at incorporating non-natural polyketide building blocks.

Our structures have provided insight into substrate binding of an isomerase that should catalyze a straightforward catalytic acid-base reaction, which has been previously compared to proline racemase. However, an essentially perfect transition state analog, binds in an orientation with the proposed catalytic acids at too far a distance for catalysis. Future studies with non-enolizable analogs and neutron diffraction studies will help clarify the roles of the active site residues.

\section{ASSOCIATED CONTENT}

\section{Supporting Information}

The Supporting Information is available free of charge on the ACS Publications

website.

Protein production, crystallization and structure solution, supplementary figures, supplementary tables and supplementatry references (PDF)

\section{AUTHOR INFORMATION}

\section{Corresponding Author}

Jeremy R. Lohman - Department of Biochemistry, Purdue University, West Lafayette, IN 47907; orcid.org/0000-0001-8199-2344; Email: jlohman@purdue.edu

\section{Notes}

The authors declare no competing financial interests.

\section{ACKNOWLEDGMENT}

We acknowledge support from Purdue University Center for Cancer Research's American Cancer Society Institutional Grant program for new investigators and Ralph W. and Grace M. Showalter Research Trust. This research used resources of the Advanced Photon Source, a U.S. Department of Energy (DOE) Office of Science User Facility operated for the DOE Office of Science by Argonne National Laboratory under Contract No. DE-AC02-06CH11357. Use of the LS-CAT Sector 21 was supported by the Michigan Economic Development Corporation and the Michigan Technology Tri-Corridor (Grant 085P1000817).

\section{REFERENCES}

1. Dobson, C. M.; Gradinger, A.; Longo, N.; Wu, X.; Leclerc, D.; Lerner-Ellis, J.; Lemieux, M.; Belair, C.; Watkins, D.; Rosenblatt, D. S.; Gravel, R. A., Homozygous nonsense mutation in the MCEE gene and siRNA suppression of methylmalonyl-CoA epimerase expression: a novel cause of mild methylmalonic aciduria. Mol Genet Metab 2006, 88 (4), $327-$ 33.

2. Gross, F.; Ring, M. W.; Perlova, O.; Fu, J.; Schneider, S.; Gerth, K.; Kuhlmann, S.; Stewart, A. F.; Zhang, Y.; Muller, R., Metabolic engineering of Pseudomonas putida for methylmalonyl-CoA biosynthesis to enable complex heterologous secondary metabolite formation. Chemistry \& biology 2006, 13 (12), 1253-64.

3. Dayem, L. C.; Carney, J. R.; Santi, D. V.; Pfeifer, B. A.; Khosla, C.; Kealey, J. T., Metabolic engineering of a methylmalonyl-CoA mutaseepimerase pathway for complex polyketide biosynthesis in Escherichia coli. Biochemistry 2002, 41 (16), 5193-201.

4. Marsden, A. F.; Caffrey, P.; Aparicio, J. F.; Loughran, M. S.; Staunton, J.; Leadlay, P. F., Stereospecific acyl transfers on the erythromycin-producing polyketide synthase. Science 1994, 263 (5145), $378-80$.

5. Peter, D. M.; Vogeli, B.; Cortina, N. S.; Erb, T. J., A ChemoEnzymatic Road Map to the Synthesis of CoA Esters. Molecules 2016, 21 (4), 517.

6. Hughes, A. J.; Keatinge-Clay, A., Enzymatic extender unit generation for in vitro polyketide synthase reactions: structural and functional showcasing of Streptomyces coelicolor MatB. Chemistry \& biology 2011, 18 (2), 165-76.

7. $\quad$ Crosby, H. A.; Rank, K. C.; Rayment, I.; Escalante-Semerena J. C., Structure-guided expansion of the substrate range of methylmalonyl coenzyme A synthetase (MatB) of Rhodopseudomonas palustris. Appl Environ Microbiol 2012, 78 (18), 6619-29.

8. Pohl, N. L.; Hans, M.; Lee, H. Y.; Kim, Y. S.; Cane, D. E.; Khosla, C., Remarkably broad substrate tolerance of malonyl-CoA synthetase, an enzyme capable of intracellular synthesis of polyketide precursors. Journal of the American Chemical Society 2001, 123 (24), $5822-3$.

9. Koryakina, I.; Williams, G. J., Mutant malonyl-CoA synthetases with altered specificity for polyketide synthase extender unit generation. Chembiochem : a European journal of chemical biology 2011, 12 (15), 2289-93.

10. Koryakina, I.; McArthur, J.; Randall, S.; Draelos, M. M.; Musiol, E. M.; Muddiman, D. C.; Weber, T.; Williams, G. J., Poly specific trans-acyltransferase machinery revealed via engineered acyl-CoA synthetases. ACS chemical biology 2013, 8 (1), 200-8.

11. Koryakina, I.; Kasey, C.; McArthur, J. B.; Lowell, A. N.; Chemler, J. A.; Li, S.; Hansen, D. A.; Sherman, D. H.; Williams, G. J., Inversion of Extender Unit Selectivity in the Erythromycin Polyketide 
Synthase by Acyltransferase Domain Engineering. ACS chemical biology 2017, 12 (1), 114-123.

12. Musiol-Kroll, E. M.; Zubeil, F.; Schafhauser, T.; Hartner, T.; Kulik, A.; McArthur, J.; Koryakina, I.; Wohlleben, W.; Grond, S.; Williams, G. J.; Lee, S. Y.; Weber, T., Polyketide Bioderivatization Using the Promiscuous Acyltransferase KirCII. ACS synthetic biology 2017.

13. Carpenter, S. M.; Williams, G. J., Extender Unit Promiscuity and Orthogonal Protein Interactions of an Aminomalonyl-ACP Utilizing Trans-Acyltransferase from Zwittermicin Biosynthesis. ACS chemical biology 2018, 13 (12), 3361-3373.

14. Kalkreuter, E.; CroweTipton, J. M.; Lowell, A. N.; Sherman, D. H.; Williams, G. J., Engineering the Substrate Specificity of a Modular Polyketide Synthase for Installation of Consecutive Non-Natural Extender Units. Journal of the American Chemical Society 2019, 141 (5), 1961-1969.

15. Fuller, J. Q.; Leadlay, P. F., Proton transfer in methylmalonyl$\mathrm{CoA}$ epimerase from Propionibacterium shermanii. The reaction of $(2 \mathrm{R})$ methylmalonyl-CoA in tritiated water. Biochem J 1983, 213 (3), 643-50.

16. Leadlay, P. F.; Fuller, J. Q., Proton-Transfer in MethylmalonylCoa Epimerase from Propionibacterium-Shermanii - Studies with Specifically Tritiated (2r)-Methylmalonyl-Coa as Substrate. Biochem J 1983, 213 (3), 635-642.

17. Michenfelder, M.; Hull, W. E.; Retey, J., Quantitative measurement of the error in the cryptic stereospecificity of methylmalonylCoA mutase. European Journal of Biochemistry 1987, 168 (3), 659-667.
18. McCarthy, A. A.; Baker, H. M.; Shewry, S. C.; Patchett, M. L.; Baker, E. N., Crystal Structure of Methylmalonyl-Coenzyme A Epimerase from P. shermanii. Structure 2001, 9 (7), 637-646.

19. Stunkard, L. M.; Kick, B. J.; Lohman, J. R., Structures of LnmK, a Bifunctional Acyltransferase/Decarboxylase, with Substrate Analogues Reveal the Basis for Selectivity and Stereospecificity. Biochemistry 2021, $60(5), 365-372$.

20. Stunkard, L. M.; Dixon, A. D.; Huth, T. J.; Lohman, J. R., Sulfonate/Nitro Bearing Methylmalonyl-Thioester Isosteres Applied to Methylmalonyl-CoA Decarboxylase Structure-Function Studies. Journal of the American Chemical Society 2019, 141 (13), 5121-5124.

21. Leadlay, P. F., Purification and characterization of methylmalonyl-CoA epimerase from Propionibacterium shermanii. Biochem J 1981, 197 (2), 413-9.

22. Heuberger, K.; Bailey, H. J.; Burda, P.; Chaikuad, A.; Krysztofinska, E.; Suormala, T.; Burer, C.; Lutz, S.; Fowler, B.; Froese, D. S.; Yue, W. W.; Baumgartner, M. R., Genetic, structural, and functional analysis of pathogenic variations causing methylmalonyl-CoA epimerase deficiency. Biochim Biophys Acta Mol Basis Dis 2019, 1865 (6), 12651272 .

23. Perkins, C. M.; Rose, N. J.; Stenkamp, R. E., The structure of cobalt methylmalonate complexes, $\mathrm{CoClN} 4 \mathrm{O} 7 \mathrm{C} 10 \mathrm{H} 28$ and CoCl2N4O5.5C11H28, models for metal complexes of $\gamma$-carboxyglutamic acid. Inorganica Chimica Acta 1990, 172 (1), 119-125. 